\title{
Manganese and Silicon Distribution between Slag and Metal in Silicomanganese Production
}

\author{
Weizhong DING and Sverre E. OLSEN ${ }^{11}$ \\ Department of Materials Science and Engineering, Shanghai University, 149 Yan Shang Road, Shanghai 200072 China. \\ 1) Department of Materials Technology and Electrochemistry, Norwegian University of Science and Technology, N-7491 \\ Trondheim Norway. E-mail: sverre.olsen@matek.sintef.no.
}

(Received on February 21, 2000; accepted in final form on May 23, 2000)

\begin{abstract}
Laboratory measurements have been carried out to investigate the equilibrium distribution of manganese and silicon between slag and metal in silicomanganese production. Graphite crucibles have been used to study equilibrium between $\mathrm{Mn}-\mathrm{Si}-\mathrm{C}_{\text {sat }}$ alloys and $\mathrm{MnO}-\mathrm{SiO}_{2}-\mathrm{CaO}-\mathrm{Al}_{2} \mathrm{O}_{3}-\mathrm{MgO}$ slags in $\mathrm{CO}$ gas at $1600^{\circ} \mathrm{C}$, $1650^{\circ} \mathrm{C}$ and $1700^{\circ} \mathrm{C}$.

The equilibrium content of $\mathrm{Si}$ in the metal is mainly controlled by the temperature, the silica content of the slag and the mass ratio $R=(\mathrm{CaO}+\mathrm{MgO}) / \mathrm{Al}_{2} \mathrm{O}_{3}$. The silicon content increases with the temperature and the silica content, and decreases with increasing $R$-ratio. The silicon content remains approximately the same when some $\mathrm{MgO}$ replaces $\mathrm{CaO}$ in the slag.

The equilibrium content of $\mathrm{MnO}$ in silicomanganese slags is primarily controlled by the temperature and the silica content of the slag. Addition of $\mathrm{Al}_{2} \mathrm{O}_{3}$ to acid slags will result in somewhat lower $\mathrm{MnO}$ contents, and addition to more basic slags has the opposite effect. The equilibrium content of $\mathrm{MnO}$ in the slag is slightly increased when some $\mathrm{CaO}$ is replaced by $\mathrm{MgO}$.
\end{abstract}

KEY WORDS: silicon distribution; manganese distribution; silicomanganese; equilibrium relations; equilibrium diagrams.

\section{Introduction}

The distribution of silicon and manganese between carbon-saturated $\mathrm{Mn}-(\mathrm{Fe})-\mathrm{Si}-\mathrm{C}$ alloys and $\mathrm{MnO}-\mathrm{SiO}_{2}-\mathrm{CaO}-$ $\mathrm{Al}_{2} \mathrm{O}_{3}-\mathrm{MgO}$ slags in equilibrium with $\mathrm{CO}$ gas is a result of simultaneous reactions taking place on production of manganese ferroalloys, especially silicomanganese. In this process the temperature may reach $1600^{\circ} \mathrm{C}$ or higher, and the composition of metal and slag is assumed to approach equilibrium. Due to its importance, and for a better understanding of the processes, equilibrium measurements have been carried out by a number of investigators. ${ }^{1-12)}$

The basic reactions between slag, metal and the gas phase are described by the following equations:

$$
\begin{aligned}
& (\mathrm{MnO})+\underline{\mathrm{C}}=\underline{\mathrm{Mn}}+\mathrm{CO}_{(\mathrm{g})} \\
& \left(\mathrm{SiO}_{2}\right)+2 \underline{\mathrm{C}}=\underline{\mathrm{Si}}+2 \mathrm{CO}_{(\mathrm{g})}
\end{aligned}
$$

where parentheses denote the slag phase and underscored the alloy phase.

The present authors have previously defined the simultaneous establishment of the two equilibria as complete slag/metal/gas equilibrium. In recent years laboratory measurements have been conducted to determine complete equilibrium in the following systems ${ }^{1-3)}$ :

(1) $\mathrm{Mn}-\mathrm{Si}-\mathrm{C}_{\text {sat }}$ alloys and slags in the systems $\mathrm{MnO}-$ $\mathrm{SiO}_{2}-\mathrm{CaO}$ and $\mathrm{MnO}-\mathrm{SiO}_{2}-\mathrm{CaO}-\mathrm{Al}_{2} \mathrm{O}_{3}$ in the temperature range $1450^{\circ} \mathrm{C}$ to $1600^{\circ} \mathrm{C}$. ${ }^{1)}$ However, the results at $1600^{\circ} \mathrm{C}$ were not sufficient for a complete description of equilibrium relations at elevated temperatures.

(2) $\mathrm{Mn}-\mathrm{Fe}-\mathrm{Si}-\mathrm{C}_{\text {sat }}$ alloys and synthetic and industrial multicomponent slags in the temperature range $1350^{\circ} \mathrm{C}$ to $1500^{\circ} \mathrm{C}^{2,3)}$

Figure 1 illustrates complete equilibrium relations between $\mathrm{Mn}-\mathrm{Fe}(10 \%)-\mathrm{Si}-\mathrm{C}_{\text {sat }}$ alloys and five component $\mathrm{MnO}-\mathrm{SiO}_{2}-\mathrm{CaO}-\mathrm{Al}_{2} \mathrm{O}_{3}-\mathrm{MgO}$ slags, with mass ratios

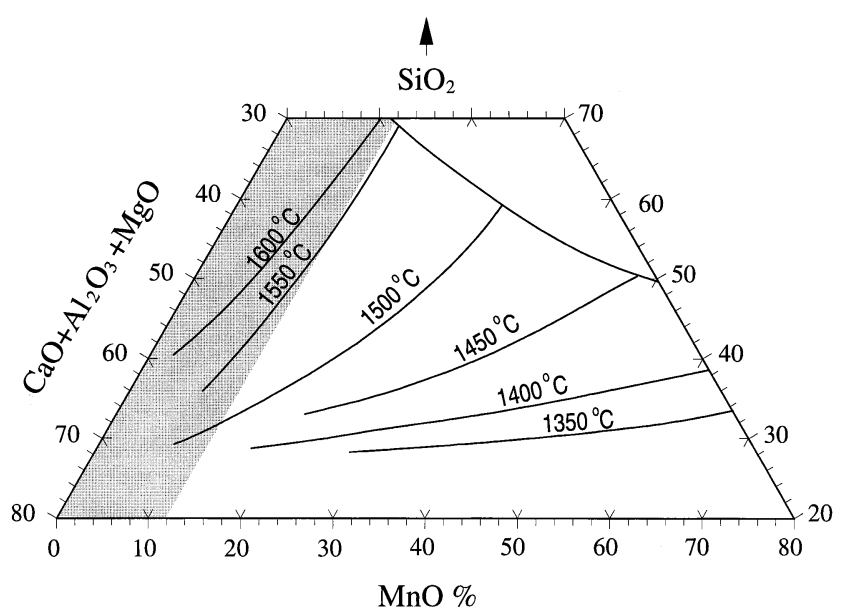

Fig. 1. Slag/metal/gas equilibrium diagram ${ }^{2)}$ for carbon saturated $\mathrm{Mn}-\mathrm{Fe}-\mathrm{Si}-\mathrm{C}$ alloys and $\mathrm{MnO}-\mathrm{SiO}_{2}-\mathrm{CaO}-\mathrm{Al}_{2} \mathrm{O}_{3}-$ $\mathrm{MgO}$ slags at $P_{\mathrm{CO}}=1 \mathrm{~atm}$, the shaded area being of interest in the present study. 
$\mathrm{CaO} / \mathrm{Al}_{2} \mathrm{O}_{3}=1.5$ and $\mathrm{MgO} / \mathrm{Al}_{2} \mathrm{O}_{3}=0.8$, at various temperatures and $P_{\mathrm{CO}}=1 \mathrm{~atm}^{2)}$ It was found that the state of complete equilibrium is very dependent on the temperature and on the pressure of the system. In contact with identical alloys, higher temperatures and higher slag basicities will give lower $\mathrm{MnO}$ contents in the slag.

A combination of the two equilibrium reactions above will give the slag/metal equilibrium reaction, expressed by

$$
2(\mathrm{MnO})+\underline{\mathrm{Si}}=2 \underline{\mathrm{Mn}}+\left(\mathrm{SiO}_{2}\right)
$$

When only the slag/metal equilibrium is established, the system is said ${ }^{1)}$ to be at partial slag/metal equilibrium. This reaction is little dependent on the temperature and independent of the pressure and composition of the gas phase. Experimental results of partial slag/metal equilibria have been reported in the literature. ${ }^{1-9)}$ It is strongly emphasized that partial slag/metal equilibrium alone will not describe completely the final composition of the melts in a carbothermic process.

High process temperatures are required for production of silicomanganese alloys by carbothermic reduction of the oxide ores. According to industrial specifications, the contents of $\mathrm{Mn}$ and $\mathrm{Si}$ in the alloy vary from 60 to $70 \%$ and from 15 to $33 \%$, respectively. Based on previous estimation, ${ }^{2)}$ the maximum $\mathrm{Si}$ content of the $\mathrm{Mn}-\mathrm{Si}-\mathrm{C}_{\mathrm{sat}}$ alloys will be about $24 \% \mathrm{Si}$ at $1600^{\circ} \mathrm{C}$ and $32 \% \mathrm{Si}$ at $1700^{\circ} \mathrm{C}$, assumed unit activity of $\mathrm{SiO}_{2}$ and a total pressure of one atmosphere, i.e. $P_{\mathrm{CO}}+P_{\mathrm{SiO}}+P_{\mathrm{Mn}}=1 \mathrm{~atm}$.

The present study focuses on complete slag/metal/gas equilibrium relations between $\mathrm{Mn}-\mathrm{Si}-\mathrm{C}_{\text {sat }}$ alloys and $\mathrm{MnO}-\mathrm{SiO}_{2}-\mathrm{CaO}-\mathrm{Al}_{2} \mathrm{O}_{3}-\mathrm{MgO}$ slags in $\mathrm{CO}$ gas (1 atm) at $1600^{\circ} \mathrm{C}$ and higher. The investigated slag compositions are limited to the low $\mathrm{MnO}$ side, as illustrated by the shaded area in Fig. 1. The equilibrium compositions are measured experimentally by using a slag-metal-gas equilibrium technique with a large metal/slag ratio.

\section{Experimental}

\subsection{Furnace Design}

A vertical furnace with a graphite heating element was used for the equilibrium measurements. The furnace assembly is shown in Fig. 2. The furnace consists of two parts, one for keeping the graphite crucible at the reaction temperature, and the other for quenching in a water-cooled jacket which allows the sample to retain its equilibrium composition.

The connection between the two parts consists of a stainless tube carrying a graphite supporter. A gas-tight motion structure is designed at the bottom of the quenching zone, so the steel tube can slide easily up and down.

The temperature profile was measured in advance. It was found that the lower position of the constant hot zone was near the bottom of the crucible. According to experience from previous measurements, ${ }^{12)}$ the temperature readings on a thermocouple in close contact with the bottom of the crucible will be $8-12^{\circ} \mathrm{C}$ lower than the real temperatures in melts. A correction had therefore to be made for this temperature difference.

Cylindrical graphite blocks were used as crucibles in all experiments. Each block had four holes acting as separate

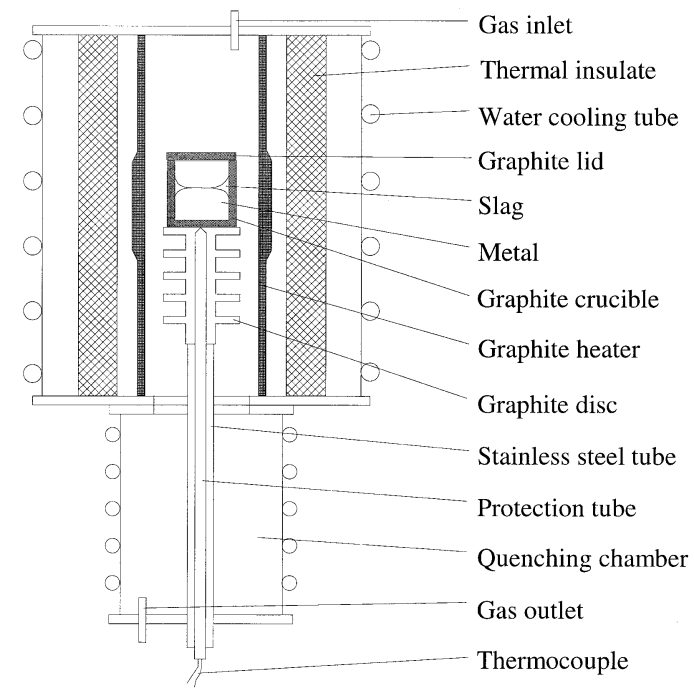

Fig. 2. Furnace arrangement.

containers. For the highest temperatures and slags with high silica contents, a deeper crucible was required. It is because the acid slags have a tendency to climb high up along the crucible wall. A graphite lid was also needed, especially at higher temperatures, to prevent soot from the top of the furnace to fall into crucibles. When the silicon content of the alloy exceeds the graphite/ $\mathrm{SiC}$ coexistence point, e.g. about $19.1 \%$ at $1600^{\circ} \mathrm{C}$, the stable carbon-containing phase is silicon carbide rather than graphite. Also in this case the graphite crucibles can be employed with safety because the inside of the graphite crucible wall will be covered with a dense layer of $\mathrm{SiC}^{12)}$

\subsection{Experimental Procedure}

Master alloys and slags were made by mixing and premelting reagent grade chemicals at desired ratios in an induction furnace. Graphite crucibles were used. Prepared alloys and slags were charged in each crucible hole, $8.5 \mathrm{~g}$ of metal and $1 \mathrm{~g}$ of slag, and the graphite crucible was placed in the hot zone of the furnace. The system was sealed and evacuated by a rotary pump. Afterwards $\mathrm{CO}$ gas was gradually filled into the furnace. The electric power should be supplied at a medium rate to avoid thermal shock on the hot parts of the furnace and massive evolution of $\mathrm{CO}$ gas from the reaction area. The temperature was controlled and monitored by a thermocouple on the outside wall of the heating element. Regular temperature calibrations were carried out by using a thermocouple underneath the crucible.

The necessary time to reach equilibrium had been determined earlier. ${ }^{12)}$ Less time was required if a large metal/slag ratio was used in the experiment. The samples were held at the reaction temperature for about $3 \mathrm{hr}$ at $1700^{\circ} \mathrm{C}$ and $5 \mathrm{hr}$ at $1600^{\circ} \mathrm{C}$. During heating, the flow rate of $\mathrm{CO}$ gas was about $15 \mathrm{l} / \mathrm{hr}$.

At the end of the run, the crucible was withdrawn immediately to the quenching zone. Metal and slag were carefully separated from the crucible, and adherent graphite was removed by grinding with sandpaper. The Si content of the metal sample was analysed by the ICP method that was checked against the conventional gravimetric method. The slag samples were analysed by electron microprobe analysis. 
The equilibrium gas composition for the system $\mathrm{Mn}-$ $\mathrm{Si}-\mathrm{C}-\mathrm{O}$ was calculated, ${ }^{12)}$ assuming unit activity of $\mathrm{SiO}_{2}$, carbon saturation, and a total pressure of one atm:

\begin{tabular}{|l|l|l|l|}
\hline Temperature & $\mathrm{P}_{\mathrm{CO}}$ & $\mathrm{P}_{\mathrm{SiO}}$ & $\mathrm{P}_{\mathrm{Mn}}$ \\
\hline $1600^{\circ} \mathrm{C}$ & 0.969 & 0.022 & 0.004 \\
\hline $1650^{\circ} \mathrm{C}$ & 0.942 & 0.050 & 0.003 \\
\hline $1700^{\circ} \mathrm{C}$ & 0.887 & 0.106 & 0.003 \\
\hline
\end{tabular}

In case of $\mathrm{SiO}$-saturation, an extensive loss of silicon to the escaping gas would be experienced due to the employed $\mathrm{CO}$ flow rate. However, a silicon balance indicated that the real Si loss was less than $0.3 \mathrm{~g}$. Then the real partial pressures were estimated to be $P_{\mathrm{SiO}}=0.0032 \mathrm{~atm}$ and $P_{\mathrm{CO}} \cong$ $1 \mathrm{~atm}$.

\subsection{Observations}

Some significant observations were made at the high temperature measurements. When experiments were carried out below $1600^{\circ} \mathrm{C}$, the cross sections of the crucibles were as shown in Figs. 3(a) and 3(b). For Si contents of the metal less than about $20 \%$, the slag covered and wetted the metal slightly better than it wetted the graphite as described by Fig. 3(a). For higher Si contents, which also means increased silica contents of the slag, the slag showed higher affinity to the crucible wall, represented by Fig. 3(b). In such cases, removal of adherent graphite from the slag was a difficult task. However, the wetting of the graphite did not violate the accomplishment of the experiments.

More trouble arose with further increase in temperature, especially for experiments with higher $\mathrm{Si}$ contents of the metal, say above $20 \%$. The higher the Si content, the higher the slag climbed on the crucible wall, see Figs. 3(c) and 3(d). In the extreme case, the inside wall of the crucible was completely covered by a thin layer of slag, and it was impossible to sample the slag for analysis. This is illustrated in Fig. 3(d). Sometimes green materials, probably $\mathrm{SiC}$ and mixtures of $\mathrm{SiC}$ and slag, were found in the upper part of the crucible, see Figs. 3(e) and 3(f). This material was not in contact with the metal and was not considered to be at equilibrium. Up to now there is no good explanation of

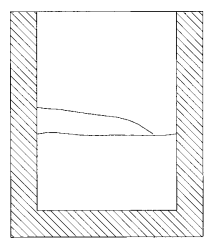

(a)

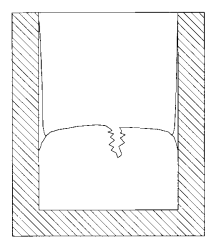

(d)

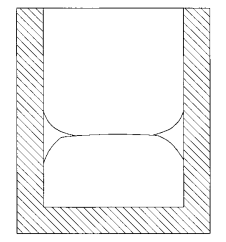

(b)

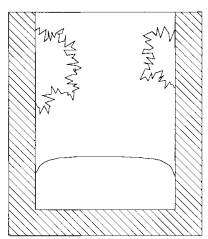

(e)

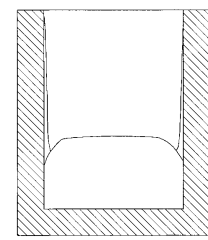

(c)

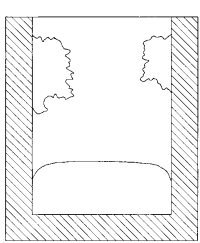

(f)
Fig. 3. Schematic cross sections of crucibles after experiments. this phenomenon.

For experiments with high $\mathrm{Si}$ contents of the metal, a rugged hole was created on the top of the solidified metal. Sometimes, after running experiments with high Si metal at high temperatures, most of the metal had escaped from the crucible.

\section{Results and Discussion}

The results of all equilibrium measurements at $1600^{\circ} \mathrm{C}$, $1650^{\circ} \mathrm{C}$ and $1700^{\circ} \mathrm{C}$ are given in Table 1. 12 experiments were carried out at $1600^{\circ} \mathrm{C}$, mostly with 4 samples in each graphite block, in total 45 samples. 24 samples from previous measurements ${ }^{1)}$ at $1600^{\circ} \mathrm{C}$ are also included at the end of the table. 9 experiments with 33 samples were run at $1650^{\circ} \mathrm{C}$, and 4 experiments with 14 samples at $1700^{\circ} \mathrm{C}$. The final $\mathrm{Si}$ and $\mathrm{Mn}$ distribution between slag and metal were determined for different mass ratios of $(\mathrm{CaO}+\mathrm{MgO}) /$ $\mathrm{Al}_{2} \mathrm{O}_{3}$ and of $\mathrm{CaO} / \mathrm{MgO}$. These oxides are not reduced to any measurable extent so the ratios remain unchanged during the experiment.

\subsection{The Equilibrium Content of $\mathrm{MnO}$ in Slag}

A typical result of complete slag/metal/gas equilibrium at $1600^{\circ} \mathrm{C}$ is shown in Fig. 4. In these experiments $\mathrm{MnO}-$ $\mathrm{SiO}_{2}-\mathrm{CaO}-\mathrm{Al}_{2} \mathrm{O}_{3}$ slags with mass ratio $\mathrm{CaO} / \mathrm{Al}_{2} \mathrm{O}_{3}=4$ were used. A minimum equilibrium content of $\mathrm{MnO}$ in the slag is observed near $44 \% \mathrm{SiO}_{2}$. Results obtained with ternary $\mathrm{MnO}-\mathrm{SiO}_{2}-\mathrm{CaO}$ slags are also shown for comparison (rhombs). Such minimum contents of $\mathrm{MnO}$ were not emphasized in previous equilibrium measurements ${ }^{1-3)}$ mostly carried out at lower temperatures, see Fig. 1.

The relation between activity and concentration of $\mathrm{MnO}$ may cause this abnormal behaviour. Figure 5 shows the activity of $\mathrm{MnO}$ in $\mathrm{MnO}-\mathrm{SiO}_{2}-\mathrm{CaO}$ slags at $1500^{\circ} \mathrm{C}$. The solid activity lines refer to pure solid $\mathrm{MnO}$ and were measured by Abraham et al. ${ }^{13)}$ The long-dash lines give a hypothetical image of the $\mathrm{MnO}$ activity in the whole region, now with pure liquid $\mathrm{MnO}$ as standard. These latter activities were calculated using the random network model by

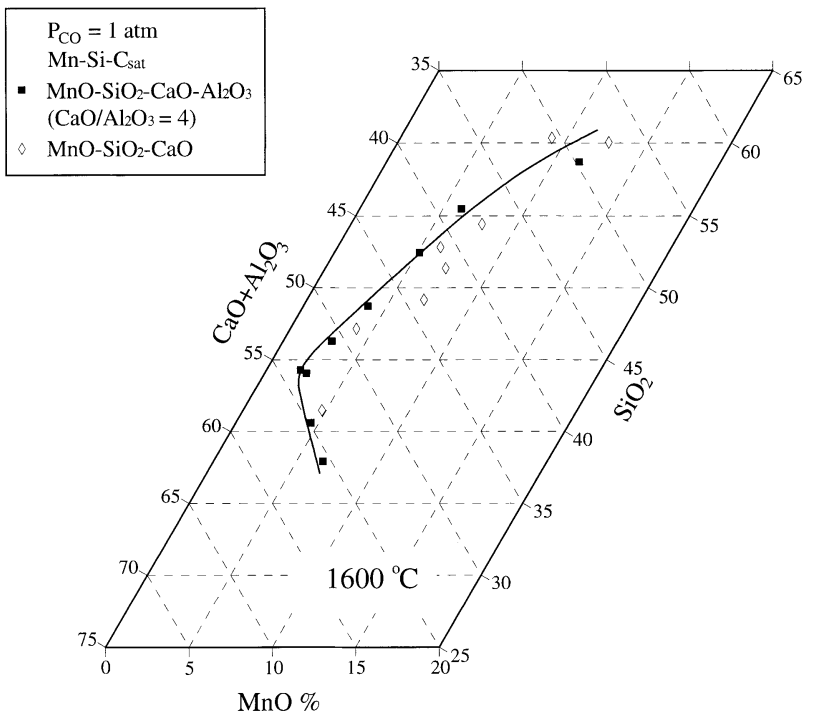

Fig. 4. Equilibrium compositions of $\mathrm{MnO}-\mathrm{SiO}_{2}-\mathrm{CaO}-\mathrm{Al}_{2} \mathrm{O}_{3}$ slags with mass ratio $\mathrm{CaO} / \mathrm{Al}_{2} \mathrm{O}_{3}=4$ at $1600^{\circ} \mathrm{C}$. 
Table 1. Experimental conditions and equilibrium compositions of $\mathrm{Mn}-\mathrm{Si}-\mathrm{C}_{\text {sat }}$ alloys and $\mathrm{MnO}-\mathrm{SiO}_{2}-\mathrm{CaO}-\mathrm{Al}_{2} \mathrm{O}_{3}-\mathrm{MgO}$ slags at various temperatures and $P_{\mathrm{CO}}=1 \mathrm{~atm}$.

\begin{tabular}{|c|c|c|c|c|c|c|c|c|c|c|c|c|}
\hline \multirow{3}{*}{$\begin{array}{c}\text { Samp. } \\
\text { No. }\end{array}$} & \multirow{3}{*}{$\begin{array}{c}\text { Temp } \\
{ }^{\circ} \mathrm{C}\end{array}$} & \multirow{3}{*}{$\begin{array}{c}\text { Time } \\
\mathrm{h}\end{array}$} & \multirow{3}{*}{$\begin{array}{l}\mathrm{M} / \mathrm{S} \\
\text { ratio }\end{array}$} & \multicolumn{3}{|c|}{ Initial composition (mass\%) } & \multicolumn{6}{|c|}{ Final composition (mass\%) } \\
\hline & & & & \multirow{2}{*}{$\frac{\text { Metal }}{\mathrm{Si}}$} & \multicolumn{2}{|c|}{ Slag } & \multirow{2}{*}{$\begin{array}{c}\text { Metal } \\
\mathrm{Si}\end{array}$} & \multicolumn{5}{|c|}{ Slag } \\
\hline & & & & & $\mathrm{SiO}_{2}$ & $\mathrm{MnO}$ & & $\mathrm{SiO}_{2}$ & $\mathrm{MnO}$ & $\mathrm{CaO}$ & $\mathrm{Al}_{2} \mathrm{O}_{3}$ & $\mathrm{MgO}$ \\
\hline D11 & 1600 & 4.5 & 5.82 & 22.32 & 62.11 & 5,17 & 21.5 & 37.98 & 2.20 & 27.82 & 32.01 & \\
\hline D12 & & & 5.03 & 16.73 & 43.16 & 2.53 & 17.2 & 27.51 & 0.75 & 36.99 & 34.75 & \\
\hline D13 & & & 6.41 & 7.00 & 35.0 & 0 & 6.2 & 28.62 & 3.28 & 34.50 & 33.60 & \\
\hline D14 & & & 5.28 & 3.53 & 35.0 & 0 & 4.9 & 30.56 & 4.95 & 32.53 & 31.96 & \\
\hline D21 & 1600 & 4.5 & 6.81 & 22.28 & 61.91 & 5.59 & 22.4 & 52.00 & 6.46 & 14.97 & 14.69 & 11.87 \\
\hline $\mathrm{D} 22$ & & & 6.24 & 16.74 & 42.46 & 3.55 & 17.2 & 43.21 & 4.17 & 19.00 & 18.33 & 15.29 \\
\hline D23 & & & 8.14 & 6.91 & 35.0 & 0 & 6.8 & 34.05 & 5.22 & 22.69 & 21.26 & 16.77 \\
\hline D24 & & & 6.37 & 3.66 & 35.0 & 0 & 3.6 & 31.57 & 6.01 & 22.80 & 21.89 & 17.64 \\
\hline D31 & 1600 & 4.5 & 6.65 & 22.56 & 61.78 & 5.97 & 21.5 & 55.49 & 6.12 & 30.47 & 7.90 & \\
\hline D32 & & & 6.07 & 16.51 & 42.5 & 5.5 & 17.3 & 44.30 & 2.07 & 42.60 & 11.04 & \\
\hline D33 & & & 7.29 & 7.06 & 35.0 & 0 & 5.9 & 40.62 & 4.53 & 43.94 & 10.91 & \\
\hline D34 & & & 6.37 & 3.55 & 35.0 & 0 & 2.8 & 37.91 & 6.59 & 44.24 & 11.26 & \\
\hline D41 & 1600 & 5.0 & 6.78 & 22.29 & 62.27 & 5.97 & 22.0 & 53.13 & 6.12 & 19.89 & 5.20 & 15.46 \\
\hline D42 & & & 6.06 & 16.49 & 43.21 & 4.20 & 17.3 & 46.81 & 3.44 & 24.58 & 6.14 & 19.03 \\
\hline D43 & & & 7.18 & 7.0 & 35.0 & 0 & 6.0 & 39.59 & 6.71 & 26.49 & 6.78 & 20.44 \\
\hline D44 & & & 7.08 & 3.68 & 35.0 & 0 & 2.8 & 36.21 & 7.39 & 27.84 & 7.08 & 21.48 \\
\hline D51 & 1600 & 5.0 & 7.57 & 24.78 & 55 & 0 & 23.6 & 59.20 & 8.71 & \begin{tabular}{|l}
17.50 \\
\end{tabular} & & 14.59 \\
\hline D52 & & & 9.54 & 25.21 & 55 & 0 & 23.9 & 61.82 & 7.44 & 15.45 & 15.29 & \\
\hline D53 & & & 7.80 & 12.17 & 40.96 & 0 & 14.9 & 36.87 & 3.30 & 30.45 & 29.39 & \\
\hline D54 & & & 8.25 & 5.77 & 35.0 & 0 & 6.6 & 31.05 & 5.23 & 32.58 & 31.14 & \\
\hline D61 & 1600 & 4.5 & 8.42 & 19.05 & 39.46 & 3.27 & 19.7 & 39.19 & 2.43 & 29.92 & 28.46 & \\
\hline D62 & & & 8.40 & 15.49 & 38.14 & 3.24 & 17.1 & 39.13 & 3.56 & 29.24 & 28.06 & \\
\hline D63 & & & 8.38 & 19.09 & 47.70 & 3.20 & 20.2 & 46.31 & 2.93 & 40.55 & 10.20 & \\
\hline D64 & & & 8.29 & 15.65 & 41.49 & 4.76 & 17.1 & 44.06 & 2.55 & 42.71 & 10.67 & \\
\hline
\end{tabular}

\begin{tabular}{|c|c|c|c|c|c|c|c|c|c|c|c|c|}
\hline \multirow{3}{*}{$\begin{array}{l}\text { Samp. } \\
\text { No. }\end{array}$} & \multirow{3}{*}{$\begin{array}{c}\text { Temp. } \\
{ }^{\circ} \mathrm{C}\end{array}$} & \multirow{3}{*}{$\begin{array}{c}\text { Time } \\
\mathrm{h}\end{array}$} & \multirow{3}{*}{$\begin{array}{l}\mathrm{M} / \mathrm{S} \\
\text { ratio }\end{array}$} & \multicolumn{3}{|c|}{ Initial composition (mass\%) } & \multicolumn{6}{|c|}{ Final composition (mass\%) } \\
\hline & & & & \multirow{2}{*}{$\begin{array}{c}\text { Metal } \\
\mathrm{Si}\end{array}$} & \multicolumn{2}{|c|}{ Slag } & \multirow{2}{*}{$\frac{\text { Metal }}{\mathrm{Si}}$} & \multicolumn{5}{|c|}{ Slag } \\
\hline & & & & & $\mathrm{SiO}_{2}$ & $\mathrm{MnO}$ & & $\mathrm{SiO}_{2}$ & $\mathrm{MnO}$ & $\mathrm{CaO}$ & $\mathrm{Al}_{2} \mathrm{O}_{3}$ & $\mathrm{MgO}$ \\
\hline D211 & \multirow[t]{4}{*}{1700} & \multirow[t]{4}{*}{3.0} & 8.51 & 6.25 & 4135 & 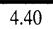 & 6.45 & 36.22 & 3.48 & 46.46 & 3.84 & \\
\hline 212 & & & 8.28 & & & & 78 & & & & & \\
\hline 5213 & & & 8.21 & 14.09 & 47.50 & 3.70 & 14.06 & 0 & 39 & 48.89 & 11.92 & \\
\hline D214 & & & 8.50 & 13.8 & 4576 & 2.72 & 14.15 & 7 & .97 & 35.75 & 33.01 & \\
\hline D221 & \multirow[t]{2}{*}{1700} & \multirow[t]{2}{*}{3.0} & 8.42 & 14. & & .46 & 3 & & & & 2 & 8.07 \\
\hline D223 & & & 8.32 & 14 & & 5.34 & 8 & 31 & & 75 & 26.08 & 12.15 \\
\hline 5241 & \multirow[t]{4}{*}{1700} & \multirow[t]{4}{*}{3.0} & 8.22 & 7.0 & 35.4 & 2.64 & 6.92 & 38 & 3.7 & 29.25 & 7.86 & 21.14 \\
\hline $\bar{D} 242$ & & & 7.85 & 20.0 & 46.1 & 2.52 & 19.18 & 41.20 & 1.2 & 36.21 & 10.05 & 11.29 \\
\hline D243 & & & 7.29 & 7.01 & & 0 & 7.37 & 31.13 & & 24.82 & 22.80 & 18.12 \\
\hline 244 & & & 7.96 & & & 2.55 & 17.60 & & & 27.20 & 26.66 & 11.72 \\
\hline 5251 & \multirow[t]{3}{*}{1650} & \multirow[t]{3}{*}{4.0} & 8.55 & & 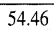 & 5.46 & 23.05 & 49.85 & 2.17 & 30.90 & 2.84 & 14.25 \\
\hline D252 & & & 8.58 & 25.2 & 52. & 5.5 & 23.77 & 48.35 & 2.4 & 33.27 & 9.15 & 6.83 \\
\hline D254 & & & 8.53 & 25. & & 5.5 & 23.88 & 41. & 2.39 & 26.87 & 27.57 & 1.42 \\
\hline D261 & \multirow[t]{4}{*}{1700} & \multirow[t]{4}{*}{3.0} & 8.62 & 19. & 45.25 & 2.75 & 17.35 & 42.38 & 0.14 & 45.26 & 11.22 & 1.00 \\
\hline D262 & & & 8.21 & 19. & & 3.41 & 19.40 & & & 36.15 & 34.37 & \\
\hline 5263 & & & 8.41 & 19. & & 0 & 18.73 & 44 & 1 & 41.54 & & 12.70 \\
\hline D264 & & & 8.27 & 10.7 & 5 & 0 & 11.12 & 43.16 & 2.74 & 32.75 & & 21.35 \\
\hline D272 & \multirow[t]{3}{*}{1650} & \multirow[t]{3}{*}{4.0} & 8.55 & 20.82 & 52.25 & 2.75 & 19.85 & 45.29 & 1.75 & 27.63 & 8.75 & 16.58 \\
\hline D273 & & & 8.47 & 16.0 & 45.64 & 5.45 & 15.52 & 43.79 & 3.14 & 27.27 & 6.63 & 19.18 \\
\hline D274 & & & 8.37 & 8.03 & 42.99 & 5.39 & 8.22 & 39.89 & 3.71 & 28.16 & 7.42 & 20.81 \\
\hline
\end{tabular}

\begin{tabular}{|c|c|c|c|c|c|c|c|c|c|c|c|c|}
\hline \multirow{3}{*}{$\begin{array}{c}\text { Samp. } \\
\text { No. }\end{array}$} & \multirow{3}{*}{$\begin{array}{c}\text { Temp. } \\
{ }^{\circ} \mathrm{C}\end{array}$} & \multirow{3}{*}{$\begin{array}{c}\text { Time } \\
\mathrm{h}\end{array}$} & \multirow{3}{*}{$\begin{array}{l}\mathrm{M} / \mathrm{S} \\
\text { ratio }\end{array}$} & \multicolumn{3}{|c|}{ Initial composition(mass\%) } & \multicolumn{6}{|c|}{ Final composition (mass\%) } \\
\hline & & & & \multirow{2}{*}{$\begin{array}{c}\text { Metal } \\
\mathrm{Si} \\
\end{array}$} & \multicolumn{2}{|c|}{ Slag } & \multirow{2}{*}{\begin{tabular}{|c|} 
Metal \\
$\mathrm{Si}$ \\
\end{tabular}} & \multicolumn{5}{|c|}{ Slag } \\
\hline & & & & & $\mathrm{SiO}_{2}$ & $\mathrm{MnO}$ & & $\mathrm{SiO}_{2}$ & $\mathrm{MnO}$ & $\mathrm{CaO}$ & $\mathrm{Al}_{2} \mathrm{O}_{3}$ & $\mathrm{MgO}$ \\
\hline D71 & 1600 & 5.0 & 8.97 & 15.19 & 39.95 & 4.63 & 16.8 & 42.23 & 4.32 & 20.02 & 18.83 & 14.60 \\
\hline D72 & & & 8.44 & 12.22 & 39.55 & 3.81 & 13.9 & 40.79 & 5.18 & 20.34 & 18.83 & 14.86 \\
\hline D73 & & & 8.55 & 9.63 & 38.7 & 6.05 & 12.0 & 39.29 & 5.95 & 20.81 & 19.07 & 14.88 \\
\hline D81 & 1600 & 4.5 & 8.52 & 26.42 & 75 & 0 & 24.0 & 72.81 & 6.32 & 10.35 & 10.53 & \\
\hline D82 & & & 8.26 & 27.12 & 70 & 0 & 4.1 & 69.99 & 7.82 & 8.25 & 7.96 & 5.98 \\
\hline D83 & & & 8.51 & 27.12 & 60 & 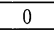 & 3.4 & 58.71 & 11.56 & 24.21 & 5.53 & \\
\hline D84. & & & 8.35 & 27.63 & 75 & 0 & 24.0 & 77.00 & 6.08 & 8.05 & 8.87 & \\
\hline D91 & 1650 & 4.2 & 8.31 & 26.13 & 64.76 & 7.48 & 24.4 & 46.35 & 3.42 & 19.58 & 18.44 & 12.21 \\
\hline D92 & & & 8.28 & 20.00 & 60.78 & 6.41 & 21.0 & 39.17 & 2.18 & 24.90 & 23.01 & 10.74 \\
\hline D93 & & & 8.23 & 17.3 & 55.53 & 6.35 & 19. & 40.46 & 2.43 & 22.52 & 20.98 & 13.61 \\
\hline D94 & & & 8.34 & 15.2 & 53.28 & 5.93 & 17.6 & 39.37 & 3.39 & 22.83 & 21.00 & 13.41 \\
\hline D101 & 1650 & 4.0 & 8.28 & 29.16 & $67 ?$ & $5 ?$ & 26.5 & 66.48 & 8.81 & 9.24 & 8.84 & 6.64 \\
\hline D102 & & & 8.48 & 19.95 & 55.74 & 5.39 & 20.4 & 36.82 & 1.54 & 31.63 & 30.02 & \\
\hline D103 & & & 8.30 & 17. & 49. & 5.34 & 19 & 38.06 & 2.57 & 30.47 & \begin{tabular}{|l}
28.90 \\
\end{tabular} & \\
\hline D104 & & & 8.66 & 15.2 & 45.86 & 5.56 & 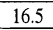 & 37.74 & 2.83 & 30.33 & \begin{tabular}{|l}
29.09 \\
\end{tabular} & \\
\hline D111 & 1600 & 5.0 & 8.59 & 14.19 & 43.23 & 5.56 & 15.7 & 46.53 & 4.62 & 24.55 & 6.17 & 18.12 \\
\hline D112 & & & 8.51 & 20.03 & 50.3 & 5.5 & 20.1 & 49.21 & 2.91 & 25.02 & 6.47 & 16.39 \\
\hline D113 & & & 8.44 & 20.04 & 52.72 & 5.45 & 19.4 & 45.22 & 4.42 & 25.42 & 24.94 & \\
\hline D114 & & & 9.5 & 19.03 & 35.0 & 0 & 10.5 & 49.56 & 3.25 & 27.33 & & 19.87 \\
\hline D121 & 1650 & 4.0 & 8.44 & 14.04 & 38.71 & 2.72 & 13.42 & \begin{tabular}{|l|l}
41.09 \\
\end{tabular} & 2.35 & 45.14 & 11.42 & \\
\hline D122 & & & 8.46 & 17.59 & 41.96 & 3.24 & 16.03 & 42.93 & 1.99 & 43.89 & 11.19 & \\
\hline D123 & & & 8.61 & 21.04 & 45.9 & 2.75 & 20.13 & 43.08 & 1.02 & 44.56 & 11.35 & \\
\hline D124 & & & 8.18 & 24.46 & 51.83 & 3.17 & 21.97 & 44.44 & 1.62 & 42.91 & 11.03 & \\
\hline D131 & 1600 & 5.0 & 8.61 & 21.06 & 52.88 & 4.44 & 19.36 & 48.75 & 3.88 & 37.59 & 9.78 & \\
\hline D134 & & & 8.64 & 20.33 & 47.63 & 4.44 & 19.59 & 46.89 & 4.97 & 17.74 & 16.94 & 13.46 \\
\hline
\end{tabular}

\begin{tabular}{|c|c|c|c|c|c|c|c|c|c|c|}
\hline \multirow{3}{*}{$\begin{array}{l}\text { Samp. } \\
\text { No. }\end{array}$} & \multirow{3}{*}{$\begin{array}{c}\text { Temperature } \\
\left({ }^{\circ} \mathrm{C}\right)\end{array}$} & \multicolumn{3}{|c|}{ Initial compositiom (mass\%) } & \multicolumn{6}{|c|}{ Final composition (mass\%) } \\
\hline & & \multirow{2}{*}{$\begin{array}{c}\text { Metal } \\
\mathrm{Si}\end{array}$} & \multicolumn{2}{|c|}{ Slag } & \multirow{2}{*}{$\begin{array}{l}\text { Metal } \\
\mathrm{Si} \\
\end{array}$} & \multicolumn{5}{|c|}{ Slag } \\
\hline & & & $\mathrm{SiO}_{2}$ & $\mathrm{MnO}$ & & $\mathrm{SiO}_{2}$ & $\mathrm{MnO}$ & $\mathrm{CaO}$ & $\mathrm{Al}_{2} \mathrm{O}_{3}$ & $\mathrm{MgO}$ \\
\hline TC-1 Ref. 1 & \multirow[t]{8}{*}{1600} & & & & 21.51 & 60.04 & 12.62 & 27.33 & & \\
\hline $\mathrm{TC}-2$ & & & & & 19.63 & 54.41 & 7.87 & 37.71 & & \\
\hline TC-3 & & & & & 17.37 & 51.36 & 7.21 & 41.42 & & \\
\hline TC-4 & & & & & 13.34 & 49.18 & 7.0 & 43.82 & & \\
\hline TC-5 & & & & & 23.90 & 60.35 & 9.07 & 30.58 & & \\
\hline TC- 6 & & & & & 20.64 & 52.83 & 6.17 & 40.99 & & \\
\hline TC-7 & & & & & 13.25 & 47.15 & 3.96 & 48.88 & & \\
\hline TC-8 & & & & & 7.34 & 41.49 & 4.74 & 53.77 & & \\
\hline QA-1 Ref. 1 & \multirow[t]{8}{*}{1600} & & & & 22.1 & 57.32 & 7.35 & 21.58 & 13.57 & \\
\hline QA-2 & & & & & 20.0 & 48.11 & 4.87 & 28.21 & 18.81 & \\
\hline $\mathrm{QA}-3$ & & & & & 16.1 & 45.85 & 2.79 & 31.84 & 19.52 & \\
\hline QA-4 & & & & & 14.7 & 41.59 & 2.17 & 34.48 & 21.73 & \\
\hline QA-5 " & & & & & 8.0 & 33.7 & 9.7 & 39.9 & 16.7 & \\
\hline QA-6 " & & & & & 1.4 & 29.5 & 5.7 & 40.9 & 23.9 & \\
\hline QA-7 & & & & & 22.0 & 61.05 & 9.04 & 18.4 & 11.51 & \\
\hline QA-8 “ & & & & & 10.75 & 36.95 & 4.79 & 36.13 & 22.13 & \\
\hline QB-1 Ref.I & \multirow[t]{8}{*}{1600} & & & & 17.38 & 45.75 & 3.06 & 38.4 & 12.75 & \\
\hline QB-2 & & & & & 9.03 & 38.03 & 2.82 & 44.67 & 14.49 & \\
\hline $\mathrm{QB}-3$ & & & & & 2.52 & 32.4 & 5.0 & 45.7 & 16.9 & \\
\hline $\mathrm{QB}-4$ & & & & & 20.52 & 50.3 & 5.37 & 33.4 & 10.9 & \\
\hline QB-5 & & & & & 1.5 & 32.3 & 4.5 & 47.1 & 16.1 & \\
\hline QB-6 " & & & & & 21.26 & 54.25 & 7.97 & 26.12 & 11.65 & \\
\hline QB-7 & & & & & 5.77 & 35.9 & 5.58 & 43.31 & 15.21 & \\
\hline QB-8 & & & & & 24.12 & 6172 & 877 & 2210 & 7.31 & \\
\hline
\end{tabular}

\begin{tabular}{|c|c|c|c|c|c|c|c|c|c|c|c|c|}
\hline \multirow{3}{*}{$\begin{array}{c}\text { Samp. } \\
\text { No. }\end{array}$} & \multirow{3}{*}{$\begin{array}{c}\text { Temp. } \\
{ }^{\circ} \mathrm{C}\end{array}$} & \multirow{3}{*}{$\begin{array}{c}\text { Time } \\
\mathrm{h}\end{array}$} & \multirow{3}{*}{$\begin{array}{l}\mathrm{M} / \mathrm{S} \\
\text { ratio }\end{array}$} & \multicolumn{3}{|c|}{ Initial composition (mass\%) } & \multicolumn{6}{|c|}{ Final composition (mass\%) } \\
\hline & & & & \multirow{2}{*}{$\begin{array}{c}\text { Metal } \\
\mathrm{Si}\end{array}$} & \multicolumn{2}{|c|}{ Slag } & \multirow{2}{*}{$\begin{array}{c}\text { Metal } \\
\mathrm{Si}\end{array}$} & \multicolumn{5}{|c|}{ Slag } \\
\hline & & & & & $\mathrm{SiO}_{2}$ & $\mathrm{MnO}$ & & $\mathrm{SiO}_{2}$ & $\mathrm{MnO}$ & $\mathrm{CaO}$ & $\mathrm{Al}_{2} \mathrm{O}_{3}$ & $\mathrm{MgO}$ \\
\hline D141 & \multirow[t]{4}{*}{1650} & \multirow[t]{4}{*}{4.0} & 8.32 & 27.63 & 58.79 & 5.87 & & 42.64 & 1.57 & 25.72 & 25.80 & 4.27 \\
\hline D142 & & & 8.29 & 27.73 & 55.53 & 5.3 & & 64 & 2.02 & 24.65 & 23.29 & 5.40 \\
\hline D143 & & & 8.49 & 10. & 36.0 & & 10.65 & 73 & 4.38 & 23.31 & 21.82 & 4.76 \\
\hline D144 & & & 8.55 & 24. & 5.2 & .93 & & 0.95 & 2.33 & 27.59 & 26.47 & 2.66 \\
\hline D151 & \multirow[t]{4}{*}{1600} & \multirow[t]{4}{*}{5.0} & 8.48 & 20.30 & 50.9 & 5. & 18.62 & 50.36 & 4.85 & 22.68 & 5.82 & 16.27 \\
\hline D152 & & & 8.53 & & 4 & 5 & 11.68 & 44.8. & 5.19 & 25.42 & 6.42 & 18.07 \\
\hline D153 & & & 8.2 & & 56.8 & 8.8 & 20.53 & 16 & 5.12 & 33.66 & 6 & \\
\hline D154 & & & 8.5 & 25.1 & 55. & D. & 20.51 & 51.60 & 6.85 & 15.40 & 14.99 & 11.17 \\
\hline D161 & \multirow[t]{4}{*}{1650} & \multirow[t]{4}{*}{4.0} & 8.50 & 24. & $J 2$. & & & 43.41 & 1.85 & 28.95 & 7.35 & 8.45 \\
\hline D162 & & & 8.49 & 24. & 49.65 & 5. & & 43.37 & 1.93 & 28.66 & 17.44 & 8.60 \\
\hline D163 & & & 8.51 & 15. & 39.2 & & & 1.69 & 2.51 & 29.57 & 17.52 & 8.71 \\
\hline D164 & & & 8.5 & & & & & & 1.66 & 29.17 & 17.68 & 8.44 \\
\hline D171 & \multirow[t]{4}{*}{1600} & \multirow[t]{4}{*}{5.0} & 8.36 & 24 & 55.7 & 5.39 & 22.60 & 53.50 & 6.22 & 21.90 & & 18.38 \\
\hline D172 & & & 8.26 & 20. & 54.8 & & 19.67 & 51.4 & 4.93 & 23.95 & & \begin{tabular}{|l|l}
19.68 \\
\end{tabular} \\
\hline D173 & & & 8.89 & 15.75 & 49.74 & 2.86 & 15.64 & 48.20 & 4.91 & 25,53 & & 21.36 \\
\hline D174 & & & 8.46 & 6.97 & 49.75 & 2.72 & 6.93 & 41.90 & 4.89 & 32.01 & & 21.19 \\
\hline D181 & \multirow[t]{4}{*}{1650} & \multirow[t]{4}{*}{$\begin{array}{l}4.0 \\
\end{array}$} & 8.33 & 29. & 65.3 & & 24.33 & \begin{tabular}{|l|l|} 
\\
\end{tabular} & 2.65 & 35.10 & 12.52 & \\
\hline D182 & & & 8.5 & 27. & & & 24.09 & 47. & 2.58 & 36.79 & 12.89 & \\
\hline D183 & & & 7.94 & & 42. & & 9.94 & 38 & 3.98 & 41.81 & 15.23 & \\
\hline D184 & & & 8.4 & 27.2 & 60.5 & 5.9 & 24.32 & 47.48 & 2.49 & 36.60 & 13.43 & \\
\hline D201 & \multirow[t]{3}{*}{1650} & \multirow[t]{3}{*}{4.0} & 8.96 & 21.11 & 49.74 & 2.86 & 20.26 & 47.94 & 2.31 & 29.72 & & 20.03 \\
\hline D202 & & & 8.74 & 7.91 & 49.55 & 4.95 & \begin{tabular}{|c|}
9.65 \\
\end{tabular} & 43.02 & 4.87 & 31.08 & & 21.04 \\
\hline D204 & & & 8.55 & 8.14 & 36.00 & 5.50 & 8.60 & 28.15 & 2.73 & 35.18 & 33.94 & \\
\hline
\end{tabular}




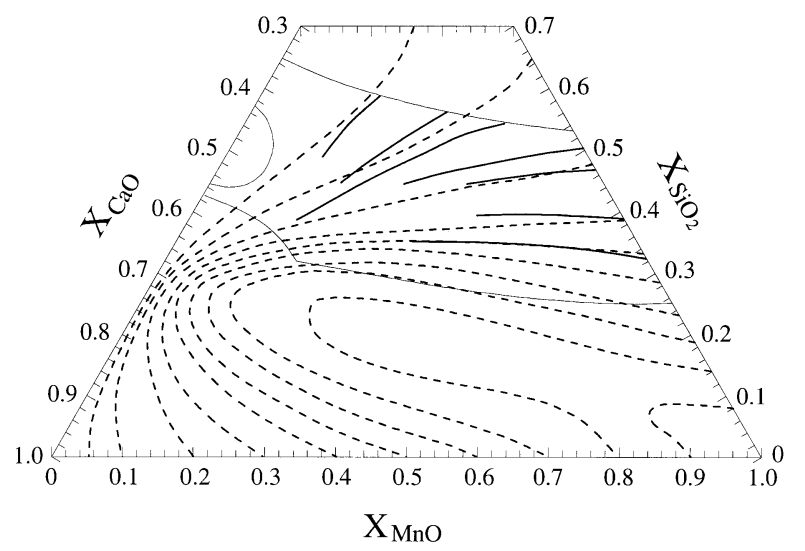

Fig. 5. Iso-activity curves of $\mathrm{MnO}$ in $\mathrm{MnO}-\mathrm{SiO}_{2}-\mathrm{CaO}$ melts at $1500^{\circ} \mathrm{C}$. The solid lines given by Abraham et al. ${ }^{13)}$ refer to pure solid MnO. The model calculated long-dashed lines refer to pure liquid $\mathrm{MnO}$.

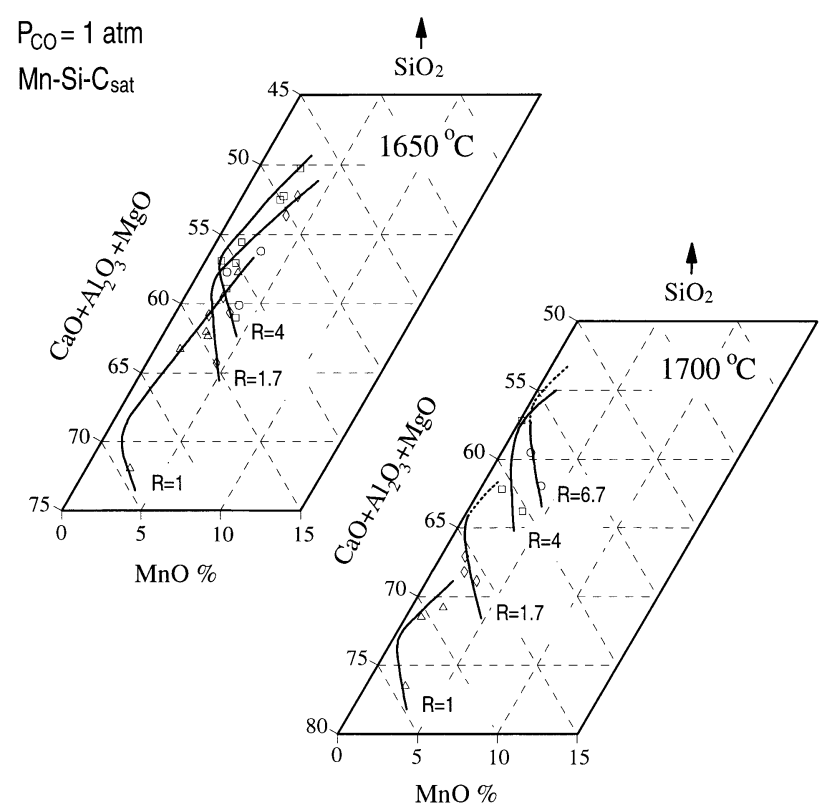

\begin{tabular}{|c|ccc|}
\hline & $\frac{\mathrm{CaO}}{\mathrm{Al}_{2} \mathrm{O}_{3}}$ & $\frac{\mathrm{CaO}}{\mathrm{MgO}}$ & $\mathrm{R}=\frac{\mathrm{CaO}+\mathrm{MgO}}{\mathrm{Al}_{2} \mathrm{O}_{3}}$ \\
\hline$\Delta$ & 1 & $\infty$ & 1 \\
$\bullet$ & 1 & 1.5 & 1.7 \\
$\square$ & 4 & $\infty$ & 4 \\
$\circ$ & 4 & 1.5 & 6.7 \\
\hline
\end{tabular}

Fig. 6. Equilibrium relations for $\mathrm{MnO}-\mathrm{SiO}_{2}-\mathrm{CaO}-\mathrm{Al}_{2} \mathrm{O}_{3}-\mathrm{MgO}$ slags with different $R$-ratios at $1650^{\circ} \mathrm{C}$ and $1700^{\circ} \mathrm{C}$. $R=$ mass ratio $(\mathrm{CaO}+\mathrm{MgO}) / \mathrm{Al}_{2} \mathrm{O}_{3}$.

Yokokawa and Niwa. ${ }^{14)}$ The activity lines indicate that the activity coefficient of $\mathrm{MnO}$ approaches a maximum near the mole ratio of $\mathrm{CaO} / \mathrm{SiO}_{2}$ equal to 2 . On the pseudobinary line $\mathrm{MnO}-\mathrm{Ca}_{2} \mathrm{SiO}_{4}$, more free oxygen ions, $\mathrm{O}^{2-}$ are released due to the strong affinity between $\left(\mathrm{SiO}_{4}\right)^{4-}$ anions and $\mathrm{Ca}^{2+}$ cations. This is quite similar to what is observed in the better known system $\mathrm{FeO}-\mathrm{SiO}_{2}-\mathrm{CaO}$.

Figure 6 shows how the complete equilibrium curves at $1650^{\circ} \mathrm{C}$ and $1700^{\circ} \mathrm{C}$ change with the value of $R^{*}$, which is defined as the ratio of the sum of basic oxides $(\mathrm{CaO}+\mathrm{MgO})$

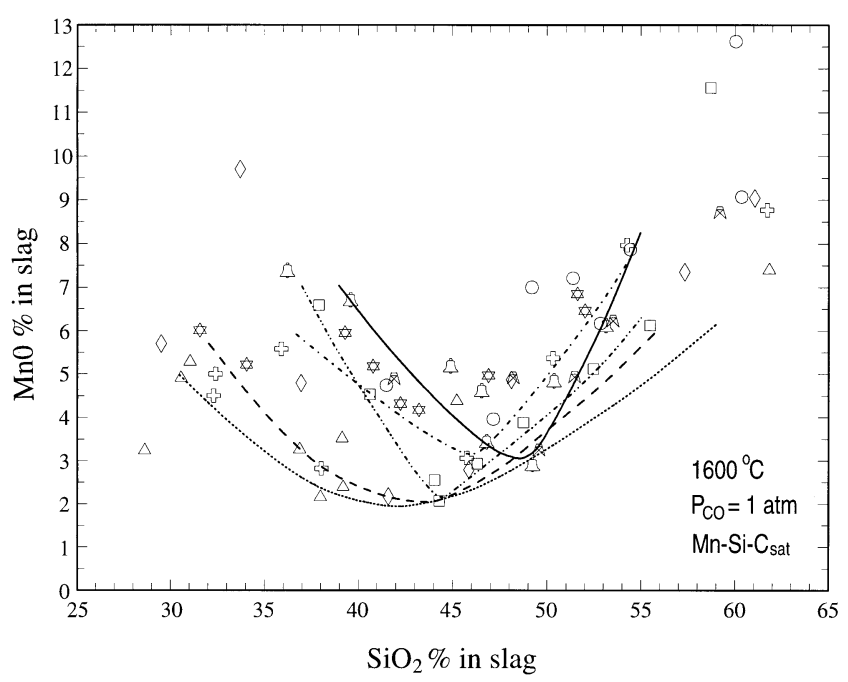

\begin{tabular}{|c|c|c|c|c|c|c|c|}
\hline & $\frac{\mathrm{CaO}}{\mathrm{Al}_{2} \mathrm{O}_{3}}$ & $\frac{\mathrm{CaO}}{\mathrm{MgO}}$ & $\mathrm{R}=\frac{\mathrm{CaO}+\mathrm{MgO}}{\mathrm{Al}_{2} \mathrm{O}_{3}}$ & & $\frac{\mathrm{CaO}}{\mathrm{Al}_{2} \mathrm{O}_{3}}$ & $\frac{\mathrm{CaO}}{\mathrm{MgO}}$ & $\mathrm{R}=\frac{\mathrm{CaO}+\mathrm{MgO}}{\mathrm{Al}_{2} \mathrm{O}_{3}}$ \\
\hline$\cdots$ & 1 & $\infty$ & 1 & $\cdots \cdots \cdots-\square$ & 4 & $\infty$ & 4 \\
\hline$\diamond$ & 1.5 & $\infty$ & 1.5 & $-\Delta$ & 4 & 1.5 & 6.7 \\
\hline$\cdots \cdots \cdot \ldots$ & 1 & 1.5 & 1.7 & 凤 & $\infty$ & 1.5 & $\infty$ \\
\hline$----\xi^{2}$ & 2.8 & $\infty$ & 2.8 & 0 & $\infty$ & $\infty$ & $\infty$ \\
\hline
\end{tabular}

Fig. 7. $\mathrm{MnO}$ versus $\mathrm{SiO}_{2}$ in multicomponent slags at $1600^{\circ} \mathrm{C}$ shown for various component mass ratios.

to $\mathrm{Al}_{2} \mathrm{O}_{3}$. This parameter has previously ${ }^{9)}$ been shown to be of considerable importance for the distribution of silicon and manganese between slag and metal. With a decrease in the $R$ value from 4 to 1 , the silica content at the minimum point drops from $43 \%$ to $31 \%$ at $1650^{\circ} \mathrm{C}$, and from $42.5 \%$ to $27 \%$ at $1700^{\circ} \mathrm{C}$.

A considerable influence of the temperature on the distribution equilibria has been verified experimentally. The equilibrium content of $\mathrm{MnO}$ in the slag decreases with increasing temperature as shown in the Figs. 1, 6 and 8. Figures 6 and 8 also indicate that the $\mathrm{MnO}$ minimum point shifts to lower silica contents with increasing temperature.

The relationship between $\mathrm{MnO}$ and $\mathrm{SiO}_{2}$ at $1600^{\circ} \mathrm{C}$ is shown in Fig. 7. Curves are drawn for $R$-values between 1 and 6.7. $\mathrm{CaO} / \mathrm{MgO}$ ratios are also given. The V-type curves get more open with a decrease in the $R$-values. Replacement of $\mathrm{CaO}$ with $\mathrm{MgO}$ shifts the curve upwards a little, and addition of more alumina shifts the curve to the left. Alumina is characterised as an amphoteric oxide. When added to acid slags, $\mathrm{Al}_{2} \mathrm{O}_{3}$ will act as a basic component and give lower $\mathrm{MnO}$ contents, and addition to more basic slags will have the opposite effect. The change between 'acid' and 'basic' slags takes place at about $45 \%$ $\mathrm{SiO}_{2}$. For a typical silicomanganese slag with about $40 \%$ $\mathrm{SiO}_{2}$ the effect of $\mathrm{Al}_{2} \mathrm{O}_{3}$ addition will be to decrease the $\mathrm{MnO}$ content somewhat in the slag.

\subsection{Distribution of Silicon between Slag and Metal}

The equilibrium distribution of silicon between $\mathrm{Mn}-\mathrm{Si}-$ $\mathrm{C}_{\text {sat }}$ alloys and $\mathrm{MnO}-\mathrm{SiO}_{2}-\mathrm{CaO}-\mathrm{Al}_{2} \mathrm{O}_{3}$ slags with mass ratio $\mathrm{CaO} / \mathrm{Al}_{2} \mathrm{O}_{3}=4$ at $1600^{\circ} \mathrm{C}, 1650^{\circ} \mathrm{C}$ and $1700^{\circ} \mathrm{C}$ is shown in Fig. 8. The measurements with high silica slags at $1700^{\circ} \mathrm{C}$ were not successful. The reason is that insufficient amounts of slag for chemical analysis were left in the cru-

* $R=$ mass ratio $(\mathrm{CaO}+\mathrm{MgO}) / \mathrm{Al}_{2} \mathrm{O}_{3}$ 


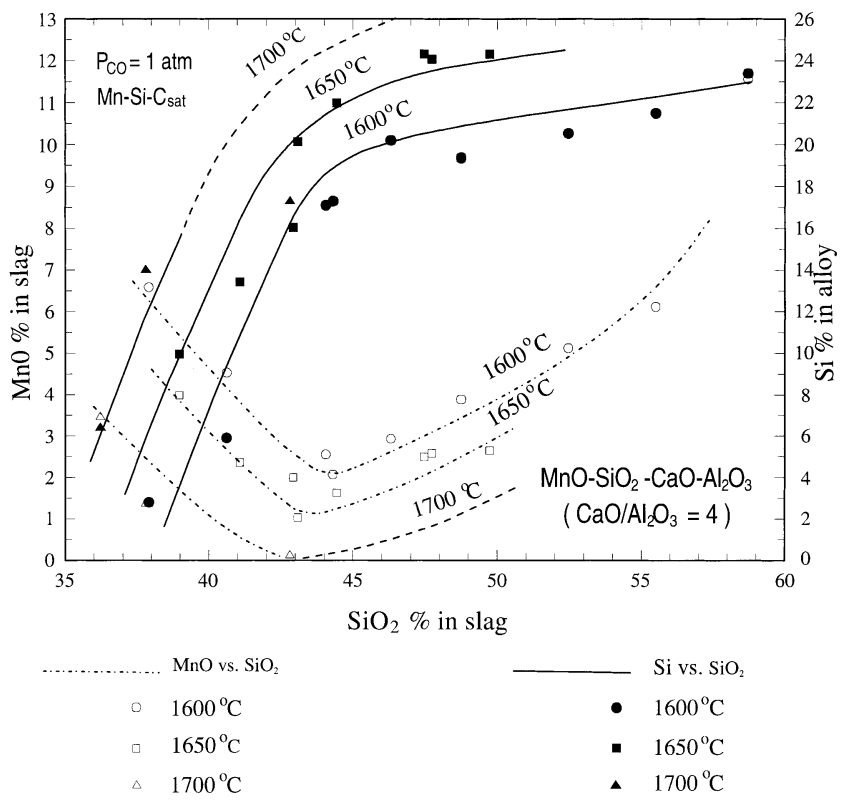

Fig. 8. Equilibrium relations for $\mathrm{MnO}-\mathrm{SiO}_{2}-\mathrm{CaO}-\mathrm{Al}_{2} \mathrm{O}_{3}$ slags with mass ratio $\mathrm{CaO} / \mathrm{Al}_{2} \mathrm{O}_{3}=4$ at $1600^{\circ} \mathrm{C}, 1650^{\circ} \mathrm{C}$ and $1700^{\circ} \mathrm{C}$.

cible. A thin layer of slag, covering the inside and outside wall of the graphite crucible, was usually observed after experiments at high temperatures and with high silica contents. The explanation is that very strong wetting between high silica slag and graphite forced the slag to climb out of the crucible.

Solid lines and marks, refer to the right y-axis, giving the silicon content of the alloy versus the silica content of the slag. The long-dashed elongation of the $1700^{\circ} \mathrm{C}$ curve indicates an expected relation. The silicon content of the alloys increases fast with increasing silica in the slag up to about $18-20 \% \mathrm{Si}$. Then follows a slow increase until silica saturation is reached. The change in slope is a result of carbon being replaced by silicon carbide as the stable carbon-containing phase. ${ }^{9)}$

The effect of temperature on the silicon distribution is considerable. As shown in Fig. 8, the equilibrium content of silicon in the alloy increases with about $6 \%$ per $50^{\circ} \mathrm{C}$ in the temperature range $1600^{\circ} \mathrm{C}$ to $1700^{\circ} \mathrm{C}$. It also appears from the figure that the equilibrium content of $\mathrm{SiO}_{2}$ in the slag, for a certain $\mathrm{Si}$-content of the metal, decreases with about $2 \%$ for an increase in the temperature of about $50^{\circ} \mathrm{C}$.

The equilibrium content of $\mathrm{MnO}$ is also shown in Fig. 8, here as chain-dashed lines and open marks. As mentioned before, the position of the MnO-minimum shows to be a function of the temperature. A comprehensive information about the complete equilibrium relations is obtained by, at the same time, reading the curves showing $\mathrm{Si} v s . \mathrm{SiO}_{2}$ and $\mathrm{MnO} v s . \mathrm{SiO}_{2}$.

The Si distribution is also dependent on other slag components and their ratios. The equilibrium distribution of silicon between metal and slag at $1600^{\circ} \mathrm{C}$ is shown for various slags at $1600^{\circ} \mathrm{C}$ in Fig. 9. The same relation is shown in Fig. 10 for the equilibrium distribution of Si at $1650^{\circ} \mathrm{C}$. The $R$-value is the major parameter determining the position of the equilibrium curves. The $\mathrm{CaO} / \mathrm{MgO}$ ratio is of minor importance.

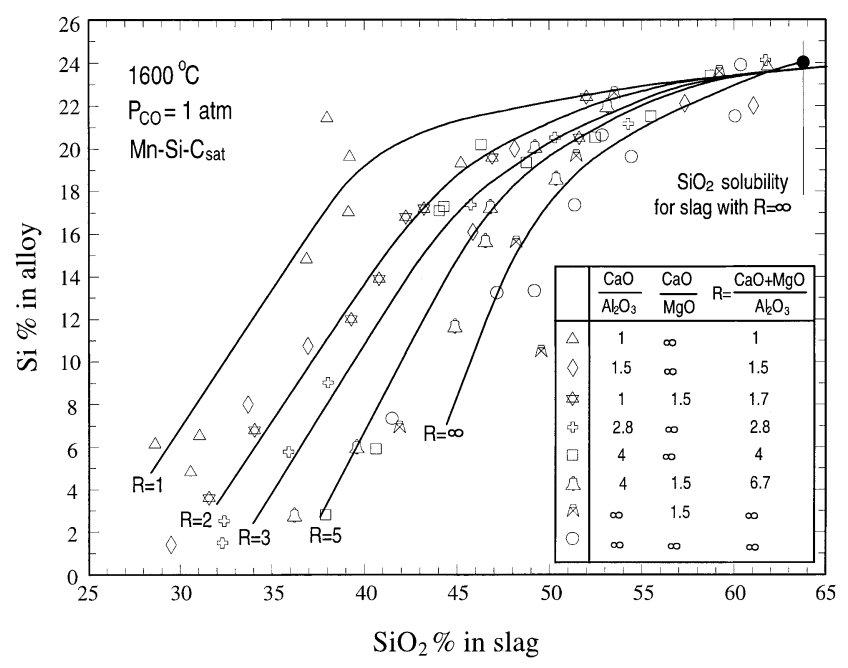

Fig. 9. Distribution of silicon as a function of the mass ratio $R=(\mathrm{CaO}+\mathrm{MgO}) / \mathrm{Al}_{2} \mathrm{O}_{3}$ at $1600^{\circ} \mathrm{C}$.

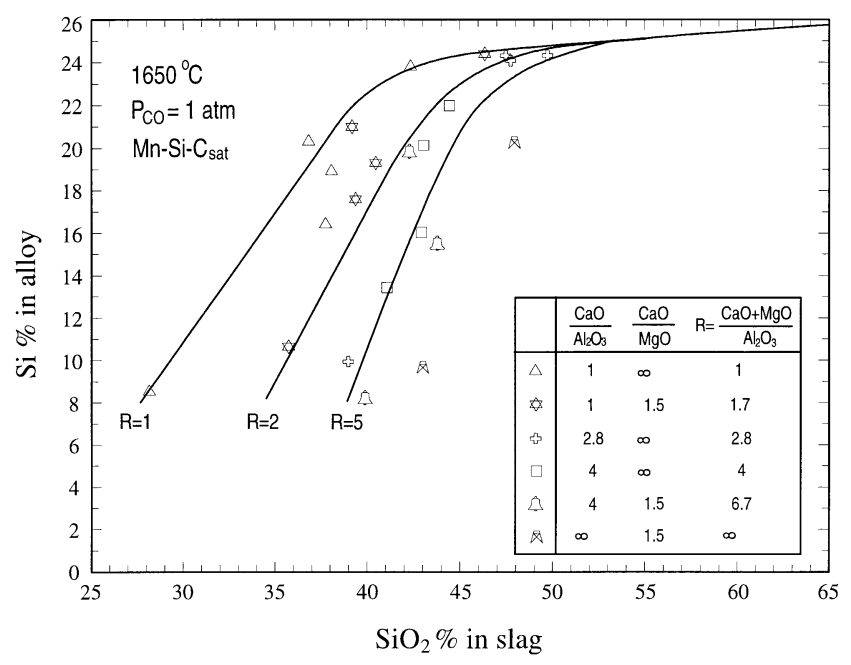

Fig. 10. Distribution of silicon as a function of the mass ratio $R=(\mathrm{CaO}+\mathrm{MgO}) / \mathrm{Al}_{2} \mathrm{O}_{3}$ at $1650^{\circ} \mathrm{C}$.

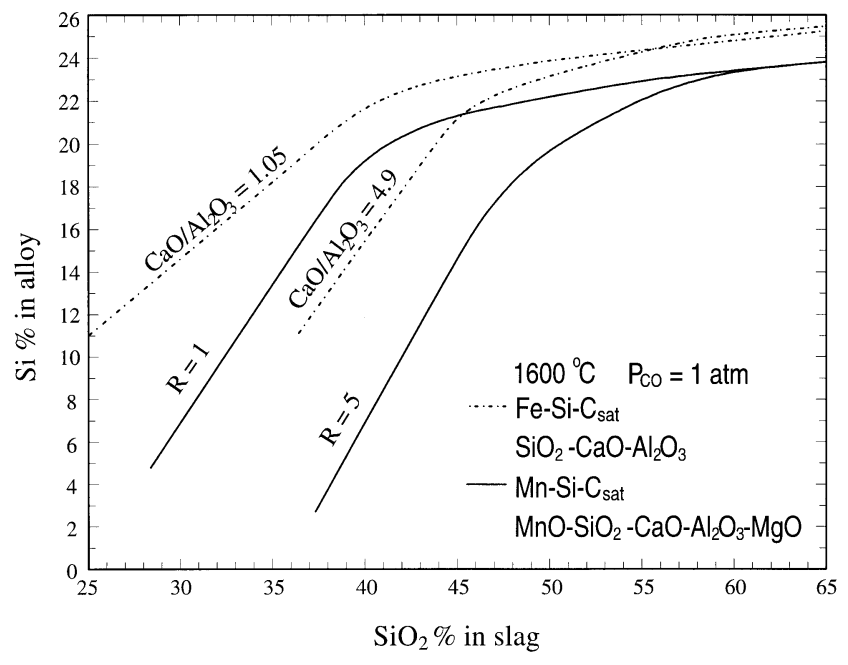

Fig. 11. Comparison of the $\mathrm{Si}$ distribution between metal and slag for $\mathrm{Mn}-\mathrm{Si}-\mathrm{C}_{\text {sat }}$ and $\mathrm{Fe}-\mathrm{Si}-\mathrm{C}_{\text {sat }}$ alloys ${ }^{15)}$ at $1600^{\circ} \mathrm{C}$. 


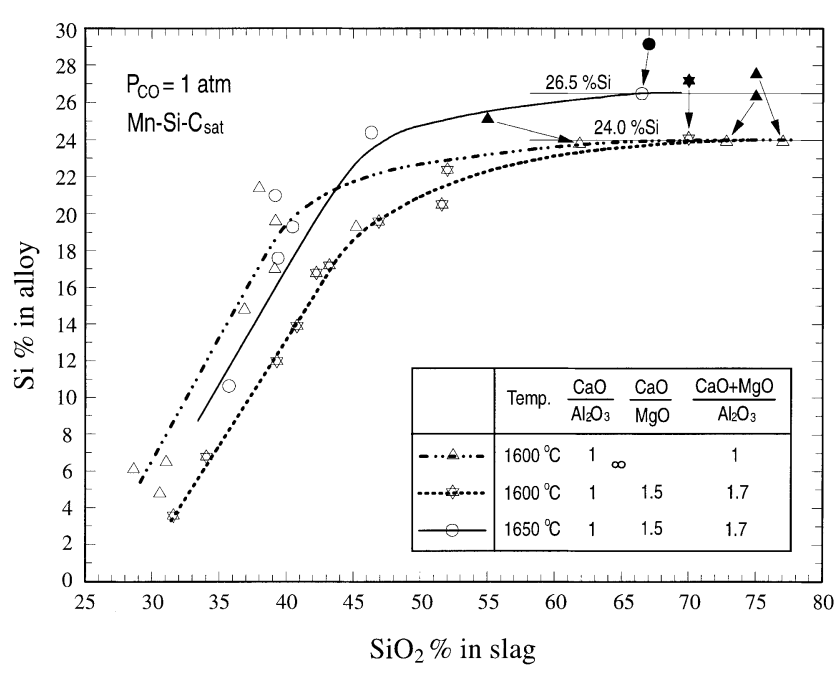

Fig. 12. Maximum Si content of $\mathrm{Mn}-\mathrm{Si}-\mathrm{Csat}$ alloys in equilibrium with silica-saturated slags at $1600^{\circ} \mathrm{C}$ and $1650^{\circ} \mathrm{C}$.

A comparison of the silicon distribution between slag and metal for $\mathrm{Mn}-\mathrm{Si}-\mathrm{C}_{\text {sat }}$ and $\mathrm{Fe}-\mathrm{Si}-\mathrm{C}_{\text {sat }}{ }^{15}$ alloys at $1600^{\circ} \mathrm{C}$ is made in Fig. 11. In principle, the difference between the two systems is that only silicon is distributed between the condensed phases in the iron system, whereas both $\mathrm{Si}$ and $\mathrm{Mn}$ are distributed in the manganese system. Otherwise the two systems are quite similar.

\subsection{The Silicon Content of Alloys Equilibrated with Silica-saturated Slags}

Figure 12 shows the the silicon content of $\mathrm{Mn}-\mathrm{Si}-\mathrm{C}_{\text {sat }}$ alloys versus the silica content of $\mathrm{MnO}-\mathrm{SiO}_{2}-\mathrm{CaO}-\mathrm{Al}_{2} \mathrm{O}_{3}-$ $\mathrm{MgO}$ slags in $\mathrm{CO}$ gas atmosphere. Solid marks indicate the starting composition of the metal melts and the arrows show the direction of approach to equilibrium. In contact with silica-saturated slags, the maximum silicon content appears to be $24.0 \% \mathrm{Si}$ at $1600^{\circ} \mathrm{C}$ and $26.5 \% \mathrm{Si}$ at $1650^{\circ} \mathrm{C}$. For comparison, the equilibrium content of silicon in $\mathrm{Fe}-\mathrm{Si}-\mathrm{C}_{\mathrm{sat}}$ alloys, also in contact with silica-saturated slag, have been reported ${ }^{15)}$ to be $26.1 \% \mathrm{Si}$ at $1600^{\circ} \mathrm{C}$.

\section{Conclusions}

The equilibrium content of $\mathrm{MnO}$ in silicomanganese slags depends first of all on the temperature and secondly on the silica content of the slag. At $1600^{\circ} \mathrm{C}$ the $\mathrm{MnO}$ content decreases from about $9 \%$ at silica saturation to a minimum of about $2-4 \%$ when the silica content is reduced to about $40-45 \%$. The equilibrium content of $\mathrm{MnO}$ in the slag is slightly increased when some $\mathrm{CaO}$ is replaced by $\mathrm{MgO}$.

Addition of $\mathrm{Al}_{2} \mathrm{O}_{3}$ to acid slags will result in lower $\mathrm{MnO}$ contents, and addition to more basic slags has the opposite effect, that is to increase $\mathrm{MnO}$ in the slag. The change between 'acid' and 'basic' slag takes place at about $45 \%$ $\mathrm{SiO}_{2}$, so for typical silicomanganese slags with about $40 \%$
$\mathrm{SiO}_{2}$, the effect of $\mathrm{Al}_{2} \mathrm{O}_{3}$ will be to decrease the equilibrium content of $\mathrm{MnO}$ somewhat in the slag.

The distribution of $\mathrm{Si}$ between $\mathrm{Mn}-\mathrm{Si}-\mathrm{C}_{\text {sat }}$ alloys and multicomponent $\mathrm{MnO}-\mathrm{SiO}_{2}-\mathrm{CaO}-\mathrm{Al}_{2} \mathrm{O}_{3}-\mathrm{MgO}$ slags is mainly determined by the temperature, the silica content of the slag and the $R$-ratio $=(\mathrm{CaO}+\mathrm{MgO}) / \mathrm{Al}_{2} \mathrm{O}_{3}$.

The effect of temperature on the silicon distribution is considerable. The equilibrium silicon content of the alloy increases with about $6 \%$ per $50^{\circ} \mathrm{C}$ in the temperature range $1600^{\circ} \mathrm{C}$ to $1700^{\circ} \mathrm{C}$.

The silicon content of the alloy increases fast with increasing silica in the slag up to about $18-20 \% \mathrm{Si}$. Then follows a slow increase until silica saturation is reached. The change in slope is a result of carbon being replaced by silicon carbide as the stable carbon containing phase. The maximum Si-content is about $24 \%$ at $1600^{\circ} \mathrm{C}$ and $26.5 \%$ at $1650^{\circ} \mathrm{C}$.

The $R$-ratio $=(\mathrm{CaO}+\mathrm{MgO}) / \mathrm{Al}_{2} \mathrm{O}_{3}$ influences strongly the $\mathrm{SiO}_{2}$-activity of the slag and thereby the $\mathrm{Si}$-content of the equilibrated alloy. As an example, an alloy in equilibrium with a slag of fixed silica content at $1600^{\circ} \mathrm{C}$ will increase its Si-content with about $8 \%$, say from 12 to $20 \%$, when the $R$-ratio is increased from 1 to 2 . The silicon content of the metal remains approximately the same when $\mathrm{MgO}$ replaces some $\mathrm{CaO}$ in the slag.

\section{Acknowledgments}

The research work has been financially supported by the Research Association of The Norwegian Ferroalloy Producers (FFF) and the Research Council of Norway (NFR). They are gratefully acknowledged.

\section{REFERENCES}

1) W. Ding and S. E. Olsen: Metall. Mater. Trans. B, 27B (1996), 5.

2) W. Ding and S. E. Olsen: Scand. J. Metall., 25 (1996), 232.

3) S. E. Olsen, W. Ding, O. Kossyreva and M. Tangstad: Proc. 7th Int. Ferroalloys Cong., The Norwegian Ferroalloy Producers Research Association, Trondheim, Norway, (1995), 591-600.

4) S. E.Olsen, W. Ding and O. Kossyreva: SINTEF Report STF24 A96530, Trondheim, Norway, (1996).

5) W. J. Rankin: Trans. Inst. Min. Metall. Sec. C, 88 (1979), C167.

6) D. R. Swinbourne, W. J. Rankin and R. H. Eric: Metall. Mater. Trans. $B, 26 B$ (1995), 59.

7) J. K. Tuset, J. Sandvik and K. Venås: SINTEF Research Report, No. 340420, Trondheim, Norway, (1979).

8) G. J. W. Kor: Metall. Trans. B, 10B (1979), 367.

9) S. E. Olsen: SINTEF Report STF24 F97617, Trondheim, Norway, (1997).

10) A. Tanaka: Tetsu-to-Hagané, 66 (1980), 1474.

11) A. Gzielo and B. Pacula: Neue Hütte, (1986), 100.

12) W. Ding: Doctoral Thesis, Norwegian Institute of Technology, (1993).

13) K. P. Abraham, M. W. Davies and F. D. Richardson: J. Iron Steel Inst., 196 (1960), 82

14) T. Yokokawa and K. Niwa: Trans. Jpn. Inst. Met., 10 (1969), 81.

15) R. H. Rein and J. Chipman: Trans. Met. Soc. AIME, 227 (1963), 1193. 